\title{
Single-Stranded RNA
}

National Cancer Institute

\section{Source}

National Cancer Institute. Single-Stranded RNA. NCI Thesaurus. Code C95939.

An RNA that consists of a single strand of ribonucleotides. Because it lacks a complementary strand this form of RNA can adopt unique tertiary structures. 